\title{
A Smart Traffic Information System
}

\author{
Medhat H. A. Awadalla \\ Department of Electrical and Computer Engineering, Sultan Qaboos University, Oman \\ Department of Communications and Computers, college of Engineering, Helwan University, Egypt
}

\begin{abstract}
Traffic congestion is a challenging problem that causes crowdedness, time waste, and sometimes harmful accidents. The paper proposes a smart traffic information system that monitors the routes and picks-up the best destination route, the least crowded route, to save time and reduce the number of accidents. The system is camera based to get the status of the different routes. The obtained video streams will be processed, analyzed, and then the determined best route will regularly be stored on a server that can be remotely accessed. The system has a web-based application that facilities its managements and provides useful information about the roads to the authorized personal. A complete prototype was implemented for this system and it was tested thoroughly.
\end{abstract}

\section{Keywords}

Traffic congestion; web-based application; smart traffic information system

\section{INTRODUCTION}

At each year, many companies manufacture around 50 million cars. This number had recorded a huge increase since 2006 until it reached more than 80 million cars in 2017. In 2017, around 881,360 cars were registered in Oman and the number is increasing annually. In 2017, around 71,212 new driving licenses were registered [1]. Due to these statistics, roads are facing an extremely dangerous war, which is traffic congestion. People are always trying to search for the best solution to avoid being in a crowded road which makes them stressful and that may cause harmful accidents. Smart traffic information system will solve this massive problem. It is a system that uses the information of the road conditions to identify if there is a congestion through crowded cars or because of an accident. It helps the user to choose the best path to go to its final destination without wasting their money or time.

The rest of this paper is organized as follows. Section 2 reviews the most relevant work to the theme of this paper. In section 3, the overall system design is presented. Section 4 gives a detailed description of system implementation and testing. Finally, section 5 concludes the paper.

\section{RELATED WORK}

According to some statistics from Royal Oman Police, it is clear that the number of the driving licenses is increasing annually. In 2011, it reached 71,212 driving licenses. This section presents the most related work implemented to solve the problem tackled by this paper.

\subsection{INIRX Crowd Sourced Traffic Data}

INIRX Crowd Sourced Traffic Data is an application used in android and iOS systems. It covers more than 160,000 miles of freeways, and highways in 126 cities across the U.S. and Canada. It uses mobile phones connected to Internet or some vehicles to analyze the crowdedness in the road. It is accurate within 2 miles per minute and the data were collected from two sources: other drivers in the INRIX Smart Driver
Network and road sensor information from departments of transportation across the country that allows the traffic maps on the INRIX traffic updated continuously. It helped to save up to 155 billion dollars for US economy [2]. However, this system actually consumes a lot of money.

\subsection{Incident Detection and Data Collection- Abacus System}

Incident detection and data collection system uses accurate cameras positioned in streets, bridges, and tunnels that detect the behavior of the cars and to identify if there is a crowd or an accident [3-5]. The captured videos will be addressed and the results will be displayed into universal XML schema. The camera records the data every 1 minute. It reports the volume, speed, occupancy and it classifies the cars into groups. The problem with this system is too expensive and it needs high quality devices to perform its process correctly.

\subsection{Beijing Real-Time Traffic Information System}

It is a system that collects and analyzes traffic information by using various methods distributed in different places to control the traffic. The information sent to a number of different end-users within BTMB (Beijing Traffic Management Bureau) [6].

It is used for different kind of roads (rounded, bridges and even highways). The system managed the traffic in Beijing during Beijing Summer Olympics 2008. The problem with this system was that it used a very advanced and expensive programs and devices. Its approximated investment is about 11 billion dollars. On the other hand, it was useful for about 18,000 buses and 650 bus lanes carrying 4.5 billion passengers and the system had one of the most advanced traffic management systems in the world. The system made a hug different for a town, which had two million drivers and over 11 million non-motorized vehicles.

\subsection{Transport Information Monitoring Environment (TIME)}

Providing citizens with an accurate information on traffic conditions can encourage journeys at times of low congestion, and an uptake of public transport. TIME project was done by Cambridge University, UK, 2011 [7]. It uses sensors (infrared detectors) and counters to indicate traffic data. This system has low price but it does not help to indicate if there is any accident. In addition, it is not that accurate compared with the other projects because it may count any movement in the road. Most cities have the types of data used in Cambridge and the developed approach could be used more extensively by Cambridge County Council and extended nationally. In addition, crowd-sourced traffic data could be used to complement the data they have obtained from sensing systems already in place. 


\section{THE PROPOSED SYSTEM DESIGN}

\subsection{System Engineering Requirements}

The proposed system will count the number of cars in 60 seconds (according to a study for Abacus) to evaluate if there is any delay in the car movement either by accidents, congestion, or any environmental effects. It should display the time in 24 hours daily without stopping. The system should give the user the right decision to take the best path by giving the user the best path that he/she can take to reach their final destination. The process of requesting and receiving data will not take a lot of time from the user. In addition, it is flexible and the user can use it anywhere.

\subsection{Design Constraints}

Many constraints limited the devices that will be used to implement the system. There were some economic constraints. It should use low cost devices with reliable performance. Therefore, its cost will be low and suitable for any user. The system should be safe for the user and the environment and it should not use any harmful components and it should be durable. In addition, it can stay for long time without being affected by surrounding conditions. Furthermore, it can be scaled and upgraded.

\subsection{Top Level Description of the Proposed System}

The proposed system has four main parts: communication method, computation devices, sensing devices, and developed software programs. SMS based system uses GSM or 3G network connection to transfer data from the user to the system and vice versa (round trip). The user sends an SMS with his location and his destination inquiring the best route to his/her destination. The PC (using some software) connected with the camera gets video frames every 60 seconds, analyses this information and sends the suggested best route to the server which in turn, sends them to the user. The proposed system block diagram and the system architecture are shown in Figure 1 and 2 respectively.

The overall system design works as shown in the sequence diagram shown in Figure 3. The user sends an SMS to the system. Then, the system gets the latest results from the file stored in the server and sends to the user. Actually, the results are obtained every 1 minute from the cameras. After that, it will be sent to the PC to be processed and analyzed. Finally, the results will be stored in the file to be sent to the user whenever he/she requests them.

The proposed system provides the following advantages:

1) Using SMS as a communication method is simple and costless because most people nowadays have mobile phones, they don't need to pay for any subscription in any service and they can use it everywhere.

2) The deployment cost is reasonable.

3) The system is automatic and user friendly.

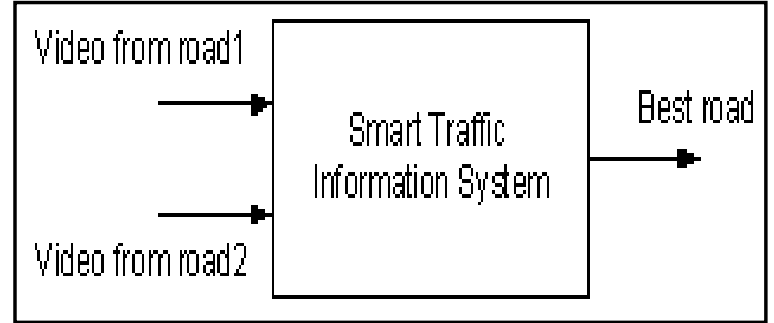

Fig.1. The proposed system block diagram

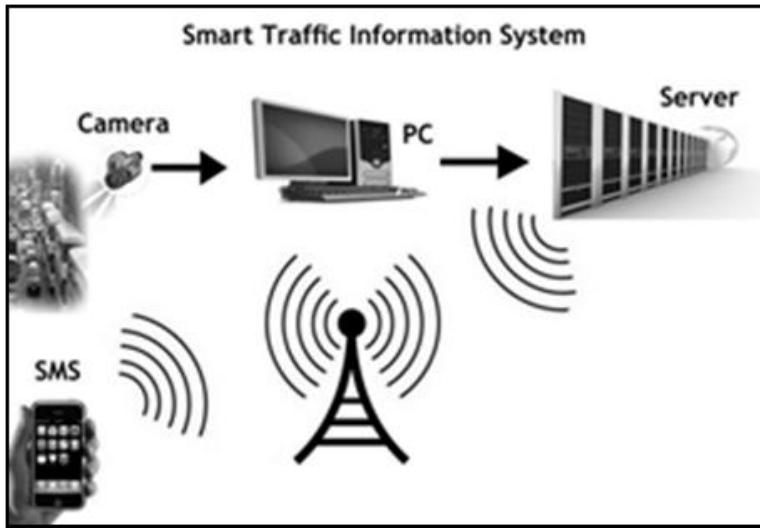

Fig. 2. The Proposed System Architecture

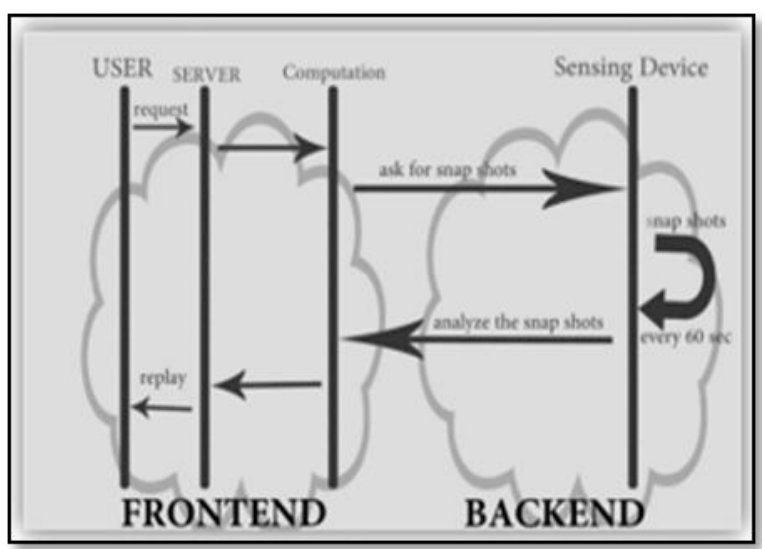

Fig. 3. The design time diagram

\section{THE DEVELOPED SOFTWARE PROGRAMS}

Image processing toolbox of MATLAB software is used in processing, analyzing and counting objects from images. It is used to detect the number of cars on the specified roads. However, it did not work efficiently because it cannot detect dark cars in the roads and it needs to eliminate all unwanted objects around the cars to change the image to a binary image consisting of zeroes and ones [8].

The optical flow in computer vision is a velocity field associated with image changes. This effect generally appears due to the relative movement between object and camera or by moving the light sources that illuminates the scene [9]. To determine the optical flow, most approaches are relying on brightness changes between two scenes. Optical flow is a command in the computer vision system toolbox. It is used to track objects in an image or a video [10]. The conducted tests showed that the system would track each car separately as it shown in Figure 4 and even for the black cars that are closely matching the background of the images as shown in Figure 5. 
The optical flow approach has been tested with 21 cars, seven of them dark colored and the approach managed to detect all of them.
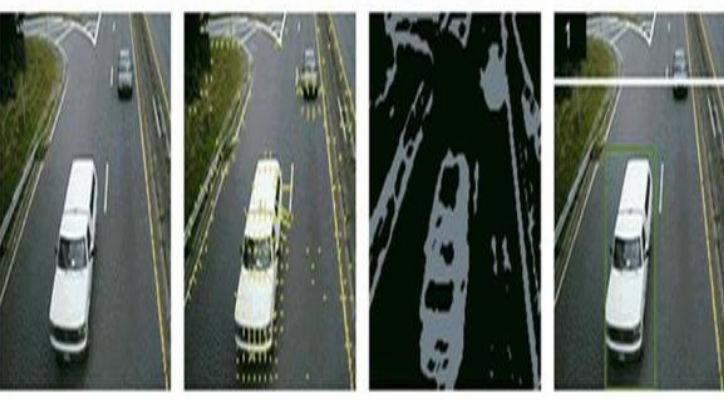

Fig. 4. The achieved optical flow results for one car category

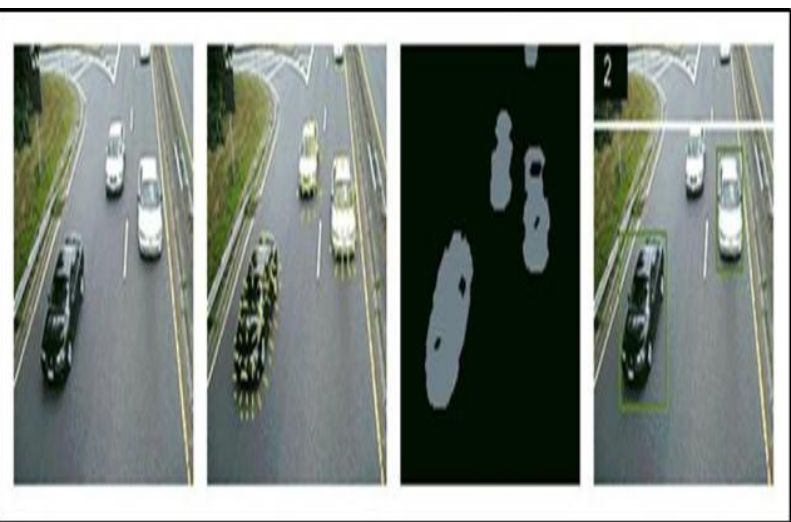

Fig. 5. Optical flow results for colored cars

\section{IMPLEMENTATION AND TESTING}

In the following sub-sections, the system modules will be addressed, implemented, and tested individually.

\subsection{Tracking and Counting System}

In general, the system detects the moving objects from a sequence of video frames that are fed to the system. The system makes threshold process to determine if the object is a real car to be considered and then counts the number of cars on each road. The output of the system is the chosen best route depending on number of cars on each road. The system comprises five sub-systems as shown in Figure 6. The subsystems are color conversion, motion detection, threshold processing, boundary boxes, and counting and these units will be described next in more details.

First, the camera will continuously take video frames. The color information of the input video frames is converted into grayscale color by changing the colors using space conversion block (from R'G'B mode to intensity mode). It is an additive way for colors red, green, and blue together to produce an array of colors with equal levels and will appear as a grey color.

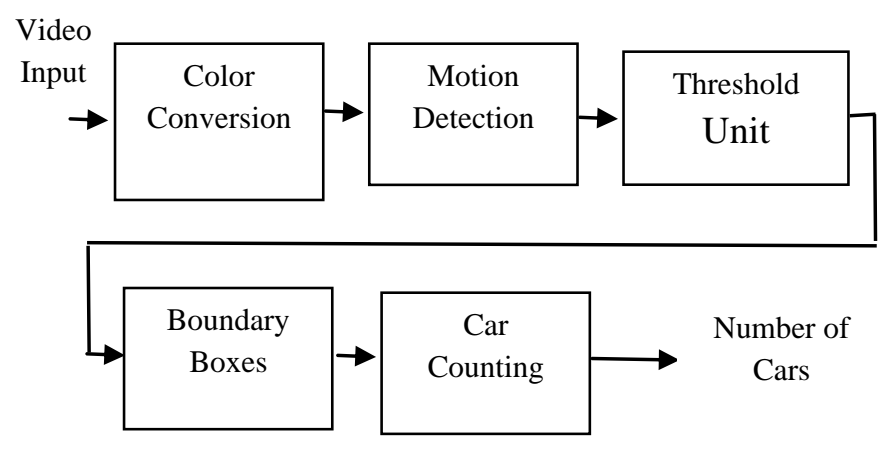

Fig. 6. The Proposed Sub-system Platform

Then, the optical flow block will estimate the motion of the object by comparing two consecutive frames. It detects the motion if there is a smooth flow that gives accurate time derivatives by using more than two frames as shown in the Figure 7.

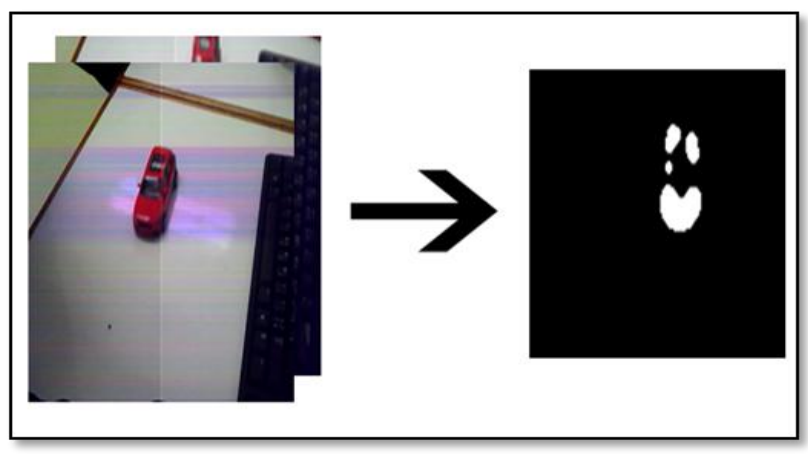

Fig.7. Estimating motion using multiple frames

At the threshold process stage as shown in Figure 8, the velocity of the movement object is considered as an input for the math function block that gets the velocity magnitude to the power of two. The output of math function is connected directly to a rational block and meanwhile to mean block to take the average velocity of two frames and then pass it to "mean 1 " for calculating the average velocity across time. The "Gain" is used to get an accurate result by reducing the noise. If the input velocity is greater than the mean value, it will be mapped to one and zero otherwise. Median filter block will take the average of the neighborhood pixels to remove noise of the images and then, pass it to the closing block for classifying frames and do some morphological closing to get clear frames [13].

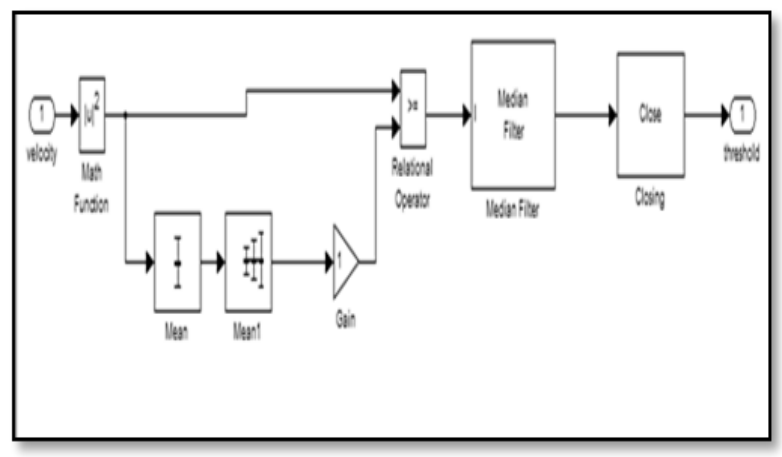

Fig. 8. The threshold sub-system 
The blob analysis block, shown in Figure 9, is used after specifying the background and the foreground of the image based on motion. The frames will be passed to the blob analysis block that makes some boundary boxes around the moving objects so, it can be easier to detect and count the number of cars.

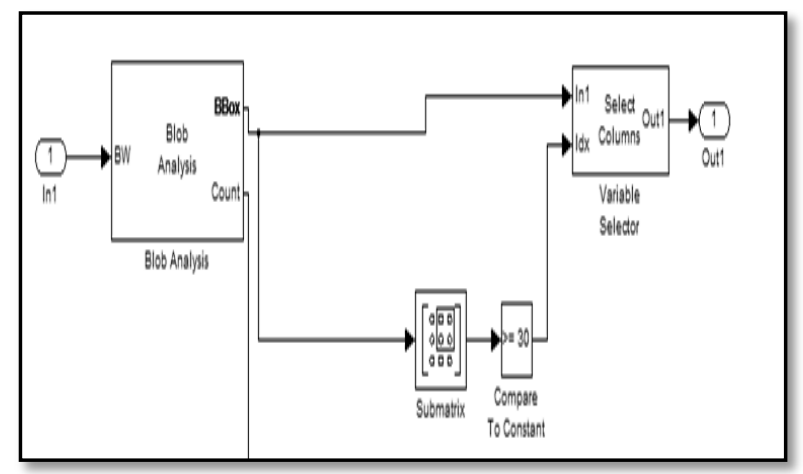

Fig. 9. Subsystem to make boundary boxes for objects
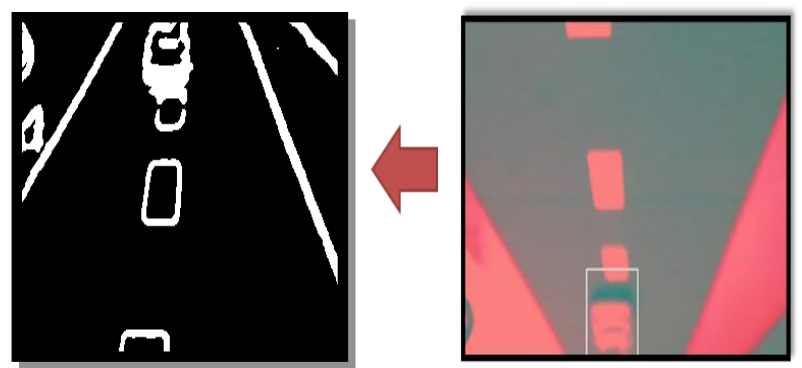

Fig. 10. Achieved results from boundary boxes subsystem

A Sub-Matrix block is added to select subset matrix from intensity matrix that enter the blob analysis block and set it to the (end column) mode. Then the output will be compared to a threshold. A "variable selector" block is added to select the wanted columns from the input matrix after it compares it to a specific threshold.

Queue block is used to store data inside a file by pushing and popping data that are coming from the blob analysis block every 1 second then the counted number is added to the previous number using Cumulative Sum block. The counted cars will be stored in a file and will be updated every 60 seconds.

The whole process that is shown in Figure 11 is to detect and count the cars based on motion.

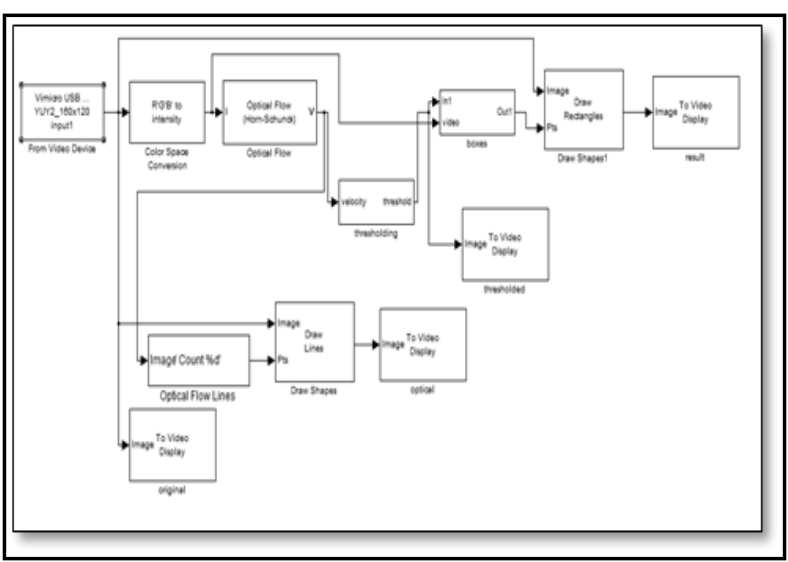

Fig 11. Detection and counting system

\subsection{GSM modem Testing and Coding with AT Commands}

The second subsystem of the design is to implement a code to receive and send the required data between the user and the system. This code was implemented using M-file in MATLAB and the communication between the modem and the MATLAB was done using AT commands [11-12].

At first, the GSM modem connectivity was tested using TMAS GSM-GPRS modem test program with the AT commands as it shown in Figure 12. Then, some commands that are responsible for receiving and reading SMSs were tested as shown in Figure 13. A "test message" was used to check the connectivity between the modem and the system.

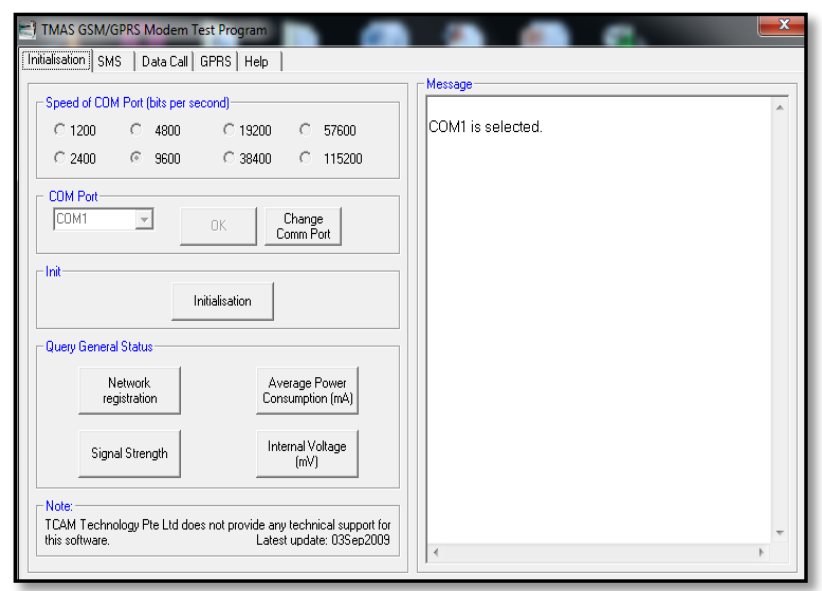

Fig. 12. Initialization of the modem

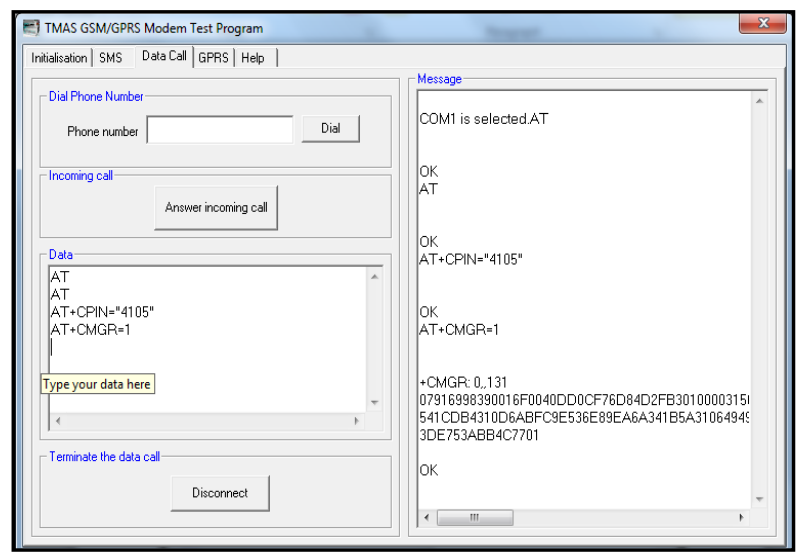

Fig. 13. Testing AT commands

\subsection{Sub-Systems Integration and testing}

At last, this code was copied into the Simulink inside the callbacks. The system was implemented to work with two different roads. The road with the least number of cars will be sent to the user when he requests it.

SQU gates were chosen to test this system. If the user sends "S-SQU", the system will analyze the data that it has to choose between to get the best path. The road with the least number of cars will be sent to the user to choose it as it is shown in Figure 14. 


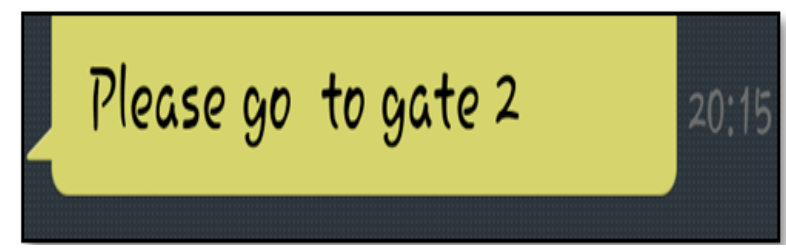

Fig. 14. The message recived from the GSM

There were multiple tests have been conducted to test the system. The first one was the unit test that was done for GSM modem as it was described earlier. The second test was the integration test. Table 1 shows the integration test results after several tests that were performed.

The complete system was tested using the model shown in Figure 15.

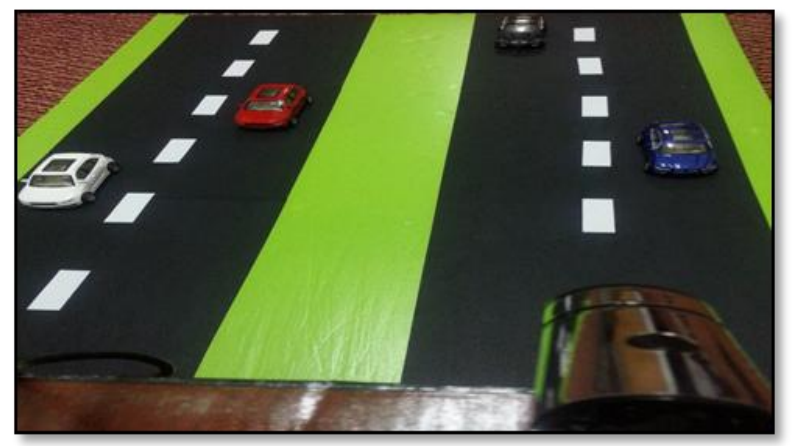

Fig. 15. The developed model of the system

Table 1. Integration test for the system

\begin{tabular}{|l|c|l|l|}
\hline Procedure & PASS & FAIL & N/A \\
\hline Tracking the cars & $\checkmark$ & & \\
\hline Counting number of cars & $\checkmark$ & & \\
\hline Saving in a file & $\checkmark$ & & \\
\hline Identify Best route & $\checkmark$ & & \\
\hline Receive the messages & $\checkmark$ & & \\
\hline Send the required data & $\checkmark$ & & \\
\hline
\end{tabular}

\section{CONCLUSION}

In this paper, smart traffic information system has been designed. The system uses cameras positioned in the entrance of the specific roads to capture video streams that reflect the status of the roads. The software programs are developed using MATLAB program. Video frames are processed and analyzed and the results are sent remotely to a file stored in a server that can be accessed by SMS to know the best route for a specific destination.

The system presented in this paper will be of a great support to assist not only the residents of the city, who are daily commuters, taxi companies and tourism authorities but also emergency vehicles such as police, ambulance and fire trucks that can be informed of the congestion locations to avoid them.

This paper can be upgraded and improved to include moving and not moving cars. Moreover, it can be used to monitor more than two roads. In addition, it is suggested as future work that the use of Morphological operators [13] may provide better option in image processing task segment.

\section{REFERENCES}

[1] Facts and Figures ROP, "Royal Oman Police, Muscat, Opened the link on June 25, 2017.

[2] J. Bak, "INRIX,". Available from URL: http://www.inrix.com/pressrelease.asp?ID=75

[3] C. C. YIK, "Vehicle tracking and speed estimation system," Faculty of computer system \& software engineering university Malaysia Pahang, 2012.

[4] A. Ahmad, M. A. K. Rizvi, N. Mohanan, Y. Z. Sait, A. S. D'Silva and M. Sadhu, "Design of an automated system for proper utilization of travel time variability of public transport system", International Journal of Applied Engineering Research vol. 9, no. 21, pp. 9391-9400, 2015.

[5] Abacus ${ }^{\mathrm{TM}} 2.0$ Incident Detection \& Data Collection," 2010. Available from URL: https://www.iteris.com/vvd.aspx?q=10147\&c=6

[6] Beijing Real-Time Traffic Information System, China 2008. Available from URL: http://www.roadtraffictechnology.com/papers/real-time.

[7] N A Trigoni, Transport Information Monitoring Environment (TIME): Event Architecture and Context Management (TIME-EACM), 2011.

[8] J. Bacon, A. Bejan, A. Beresford, D. Evans, R. Gibbens, and K. Moody Using real-time road traffic data to evaluate congestion". Available from URL: http://www.cl.cam.ac.uk/research/time/Home/pressrelease.

[9] R. T. Kelson, M. AiresAndre Santana, and A.D. Adelardo Medeiros. Optical flow using color information: preliminary results. SAC'08 March 16-20, 2008, Fortaleza, Ceara, Brazil.

[10] B. A. Hwang, "Recorded wiebners: Computer vision and Video Processing toolbox," 2010. Available from URL: http://www.mathworks.com/webex/recordings/compvisio n_031610/index.html.

[11] Definition of GSM. Available from URL: http://www.pcmag.com/encyclopedia_term/0,1237,t=GS M\&i=43985,00.asp

[12] GSM modem AT commands" Available from URL: http://www.smsiseasy.com/technicalinfo.html \# at commands.

[13] A. Ahmad. "Morphological operators and image segmentation using MATLAB, Workshop on digital processing and its applications, Feb. 10-12, 2014, Oman

\section{AUTHOR'S PROFILE}

Medhat Awadalla is an Assistant professor at Electrical and Computer Engineering Department, Sultan Qaboos niversity. On leave from Helwan University, Egypt. He obtained his $\mathrm{PhD}$ from university of Cardiff, UK. MSc and BSc from Helwan University, Egypt. His research interest includes cloud computing, sensor networks, high performance computing and real time systems. 